\title{
Kernos
}

Revue internationale et pluridisciplinaire de religion grecque antique

2| 1989

Varia

\section{Chronique des rencontres scientifiques, des expositions, des conférences}

André Motte et loannis Loucas

\section{(2) OpenEdition}

\section{Journals}

Édition électronique

URL : http://journals.openedition.org/kernos/254

DOI : $10.4000 /$ kernos. 254

ISSN : 2034-7871

Éditeur

Centre international d'étude de la religion grecque antique

Édition imprimée

Date de publication : 1 janvier 1989

Pagination : 247-257

ISSN : 0776-3824

Référence électronique

André Motte et loannis Loucas, «Chronique des rencontres scientifiques, des expositions, des conférences », Kernos [En ligne], 2 | 1989, mis en ligne le 03 mars 2011, consulté le 16 septembre 2020. URL : http://journals.openedition.org/kernos/254 
Kernos, 2 (1989), p. 247-257.

\title{
CHRONIQUE DES RENCONTRES SCIENTIFIQUES, DES EXPOSITIONS ET DES CONFÉRENCES
}

\author{
par André MOTTE et Ioannis LOUCAS
}

De manière à pouvoir informer à temps les lecteurs de la revue, la Rédaction souhaite être avertie, aussitôt que possible, des manifestations projetées; elle se fera un plaisir de les annoncer dans cette rubrique.

\section{A. Rencontres scientifiques}

\section{Rencontres récentes}

Du 18 au 20 avril 1988 s'est tenue, à Liège, la Deuxième Rencontre égéenne internationale sur le thème Le monde égéen du Bronze moyen au Bronze récent. Sur les trente-deux communications présentées, quelquesunes intéressaient la religion. Les Actes de ce colloque sont publiés dans la revue Aegaeum. Annales d'archéologie égéenne de l'Université de Liège, volume $\mathrm{n}^{\circ} 3$, à paraître en avril 1989. Renseignements : R. LAFFINEUR, Univ. de Liège, Place du XX-Août, 32, B - 4000 LIËGE.

Du 4 au 7 juin 1988 s'est tenu, à Lyon, un colloque organisé par la Maison de l'Orient sur le thème L'espace sacrificiel dans l'antiquité classique. Une quarantaine de communications ont été présentées. Le détail en sera donné à la faveur de la publication des Actes. Renseignements : Institut d'archéologie, Maison de l'Orient, 1, rue Raulin, F - 69007 LYON.

Du 11 au 13 juin 1988 s'est tenu à Athènes le IVe Symposium international de l'Institut suédois sur le thème Celebrations of Death and Divinity in the Bronze Age Argolid. Les communications suivantes ont été présentées : B. BERGQUIST, Primary or Secondary Temple Function : The Case of Halieis; C. BOULOTIS, Synnaoi theoi : A Cult Phenomenon of the Aegean Late Bronze Age; M.E. CASKEY, Thoughts on Early Helladic Hearths; W.C. CAVANAGH, C. MEE, The Location of Mycenaean Chamber Tombs in the Argolid; M.K. DABNEY, J.C. WRIGHT, Mortuary Customs, Palatial Society and State 
Formation in the Aegean Area : A Comparative Study; K. DEMAKOPOULOU, The Burial Ritual in the Tholos and Chamber Tombs at Kokla, Argolis; B. EDER, The Dorian Migration : Religious Consequences in the Argolid; R. HÄGG, The Role of Libations in Mycenaean Ceremony and Cult; M.H. JAMESON, Perseus : The Hero of Mykenai; K. KILIAN, Patterns in the Cult Activity at Haghia Triada (Klenies), Prophitis Elias Cave (H. Adrianos) and on the Acropolis of Tiryns; I. KILIAN-DIRLMEIER, Remarks on Nonmilitary Functions of Swords in Mycenaean Times; R. LAFFINEUR, Grave Circle $A$ in Mycenae : Further Reflections on its History; V. LAMBRINOUDAKIS, The Mycenaean Cult in the Sanctuary of Apollo Maleatas on the Kynortion Hill; N. MARINATOS, Celebrations of Death and the Symbolism of the Lion Hunt; C. MORRIS, In Pursuit of the White-tusked Boar : Aspects of Mycenaean Hunting; W.D. NIEMEIER, Cult Scenes on Gold Rings from the Argolid; G. NORDQUIST, Middle Helladic Burial Rites in the Argolid; O. PELON, Les tombes à tholos d'Argolide : architecture et rituel funéraire; E. PROTONOTARIOU-DEILAKI, Burial Rites in the Prehistoric Argolid; D.J. PULLEN, Early Helladic Burials at Asine and Early Bronze Age Mortuary Practices; H. TZAVELLA-EVJEN, The Ring of the Queen. The 'Thirsty Demons' and their Role in the Cult of the Goddess; B. WELLS, Death at Dendra. On Mortuary Practices in a Mycenaean Community; C. ZERNER, Ceramics and Ceremony : Pottery and Burial in the Middle Bronze Age Argolid. Des Actes seront publiés. Pour tout renseignement: Swedish Institute at Athens.

Du 17 au 21 août 1988 s'est tenu à Bordeaux le XIIe Congrès de l'Association Guillaume Budé dont le thème principal était Les écrivains et le sacré. En séance plénière, F. JOUAN a fait un exposé général sur Les écrivains et le sacré en Grèce. Dans la commission de grec ont été présentées les communications suivantes : M. ALEXANDRE, Constitution d'un lexique du sacré dans la Septante; D. AUBRIOT, Le thambos dans l'Iliade; J. AXER, Le sacrifice de Clytemnestre dans l'Agamemnon d'Eschyle; F. BADER, La langue des dieux en grec; J. BERNARDI, Un poète mystique du IVe siècle de notre ère : Grégoire de Naziance; J. BOMPAIRE, Le sacré dans les discours d'Aelius Aristide; A. CHEYNS, La notion de sebas dans la poésie homérique; B. FLUSIN, La montée vers le divin dans l'Échelle du Paradis de Jean Climaque; A. GARZYA, La divinité et l'homme chez Théognis; M. LEFKOVITZ, Euripide et les dieux; H. LLOYD-JONES, Les Érinyes dans la tragédie grecque; A. MOTTE, L'expression du sacré chez Platon; J. RUDHARDT, Le sentiment $d u$ sacré chez les orateurs attiques; F. RODRIGUEZ-ADRADOS, La mantique et les chours de l'Agamemnon d'Eschyle; Y. VERNIÈRE, Les deux pôles du sacré chez Plutarque; F. VIAN, Nonnos et l'effacement du sacré; A. WARTELLE, Le vocabulaire du sacré 
chez les Pères Apologistes; M. WORONOFF, Initiation et sens du sacré en Grèce. Plusieurs communications présentées dans la commission des jeunes concernaient aussi le domaine grec : V. BOUDON, Le songe de Galien; M.-L. FREYBURGER, Le vocabulaire des institutions religieuses chez Dion Cassius; V. FROMENTIN, Denys d'Halicarnasse et les mythes grecs; C. MAUDUIT, Relations entre le sacré et le sauvage dans la tragédie; M.-P. NOËL, Le sacré chez les sophistes; A.-M. OLSCHLÄGER, Le culte de Cybèle; E. OUDOT, Le roman grec et le sacré; V. PIRENNE-DELFORGE, Conception et manifestations du sacré dans l'Hymne homérique à Aphrodite; J. SCHNÄBELÉ, Le vocabulaire des institutions religieuses chez Denys d'Halicarnasse. Autres commissions : latin (rapport présenté par J.-C. Fredouille), français, pédagogie, vigne et vin, antiquités régionales. Les communications seront publiées, sous forme de résumés, dans le Bulletin de l'Assocation G. Budé. Secrétaire général du Congrès : prof. J. JOUANNA (Paris IV), 8, rue Corot, F - 75016 PARIS.

Du 18 au 21 août 1988 s'est tenu, à Tripolis et en d'autres villes d'Arcadie, le IIIe Congrès international de l'Académie arcadienne. Dans la septantaine de communications présentées, treize concernaient directement

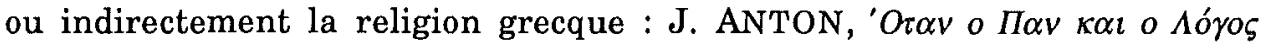

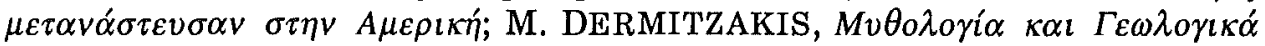

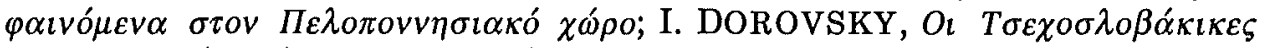

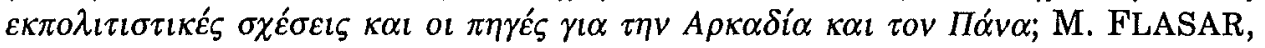

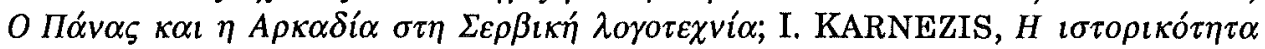

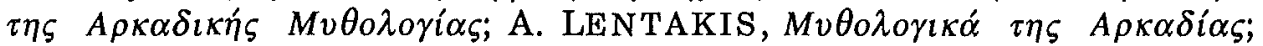

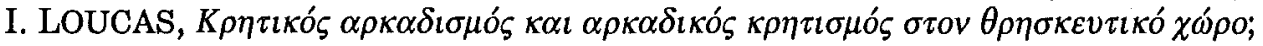

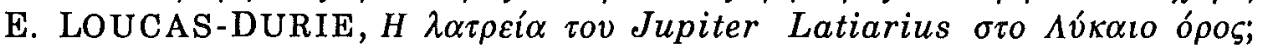

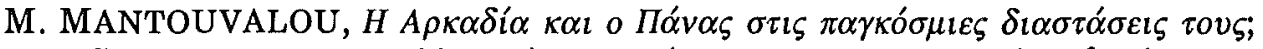

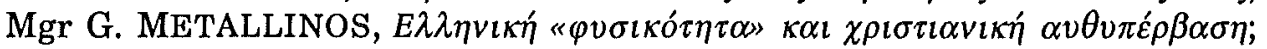

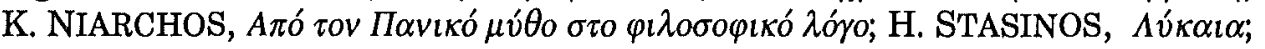

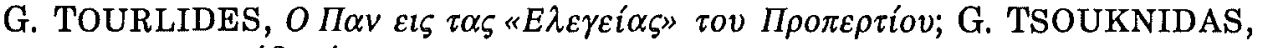

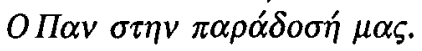

Du 25 au 30 août 1988 s'est tenu, à Paris et à Delphes, le premier Congrès international de mythologie et de psychothérapie organisé par la Société de Recherches Psychothérapiques de Langue Française et le Centre de Recherches Mythologiques de l'Université de Paris X-Nanterre, avec le concours du Centre Culturel Européen de Delphes. Le thème Angoisse et Divination y a fait l'objet de huit tables rondes. Parmi la quarantaine de communications présentées, plusieurs titres retiennent l'attention : 
R. BLOCH, Présages et prodiges antiques : déterminisme et liberté; F. JOUAN, Un fondateur de dynastie : le devin Mélampous; A. MOREAU, L'angoisse de Cassandre; C. BACONICOLA-GHORGOPOULOU, L'oracle et la dimension oraculaire du langage humain dans la Médée d'Euripide; J.-M. BATAILLE, Le complexe de Médée; J.-F. BERNARD, Le rêve de Jocaste; J. CAMBITSIS, Quelques observations sur le mythe des Protéides; M. CASEVITZ, Les rêves d'un sophiste dévot d'Asclépios : les Discours sacrés d'Aelius Aristide; B. DEFORGE, Origine mythique des plantes de vie; M.-A. DESCAMPS, Le rêve d'incubation chez les Grecs; G. ERIKSON, Le passage de l'enthousiasme à l'extase dans le monde gréco-romain; R. GELLMAN, Les religions de la préhistoire : les signes, les symboles et la sexualité; E. KOURTSIS et D. SI AHMED, Résurgence de la divination inductive dans le bassin méditerranéen; R. MARTIN, Pindaric Poetry and the Therapy of the Word : the Poet as a Mythic Healer; J.-M. MATHIEU, Bonnes et mauvaises techniques de réponse à l'angoisse chez les Pères de l'Église; E. MOUTIN-DERVAUX, Le retour à Épidaure ou la guérison éternelle; J. PEIGNEY, Le médecin et le devin : leur parenté dans la mentalité antique; C. SALLES, L'angoisse des imprécateurs : les Tabellae Defixionum; R. TRIOMPHE, Sur quelques formes végétales du sacré. Aucune information n'a encore été communiquée concernant la publication éventuelle d'Actes du colloque. Renseignements : Prof. F. JOUAN, Université de Paris X, F-92001 NANTERRE Cédex.

Du 27 au 31 août 1988 s'est tenu à Samos le Ier Congrès international sur la philosophie grecque organisé par l'Association internationale de Philosophie grecque. Parmi la septantaine de communications présentées, un grand nombre intéressaient directement ou indirectement la religion grecque.

Du 5 au 10 septembre 1988 s'est tenu, à Louvain-la-Neuve, le IVe Congrès organisé par la Société internationale des Études coptes. Des Actes sont prévus. Renseignements : Prof. J. RIES, Collège Érasme, B - 1348 LOUVAINLA-NEUVE.

Du 23 au 26 septembre 1988 s'est tenu, à Kalamata-Messène, un colloque international sur le thème Montaigne et la Grèce à l'occasion du quatrième centenaire de l'édition des Essais. Deux communications avaient rapport à la religion grecque antique : $\mathrm{A}$. MOREAU, Montaigne et les héros de la 
mythologie grecque et A. MOTTE, Montaigne et les dieux antiques. Des Actes sont prévus. Organisation: Prof. E. MOUTSOPOULOS et Prof. K. CHRISTODOULOU, Chaire de littérature française, Université d'Athènes, 27, rue Alexandroupoléos, GR-115 27 ATHÈNES.

Du 2 au 7 octobre 1988 s'est tenu, à Athènes, un séminaire sur la religion grecque organisé par les Instituts nordiques d'Athènes. Intervenants : $O$. ANDERSEN, L. BERCZELLY, S. DES BOUVRIE, J. BRAARVIG, T. EIDE, T. Frost, R. HÄGG, T. HAUKEN, D. HELLHOLM, M. HEUCHBERG, A. HULTGARD, E. KRAGGERUD, J.C. MEYER, H. MONTGOMERY, H. MOYNES, R. SKARSTEN, H. WINDFELD-HANSEN, E. WYLLER.

Du 11 au 14 novembre 1988 s'est tenu, à Tégée et à Tripolis, le IIe Colloque d'Études arcadiennes organisé par la Société d'Études péloponnésiennes et la Ligue de Tégée. Parmi les septante communications présentées, six concernaient directement ou indirectement la religion grecque : M. JOST,

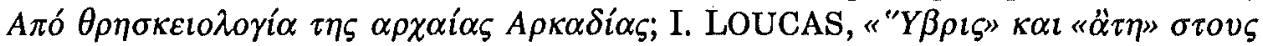

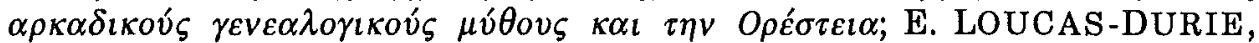

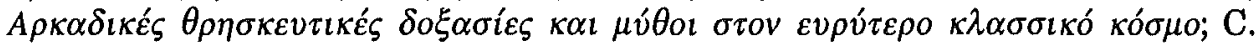

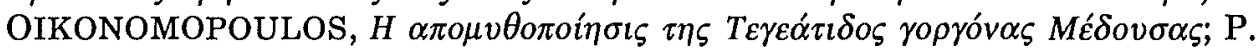

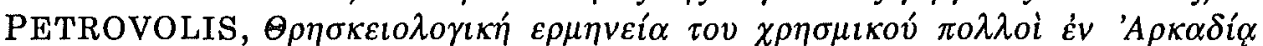

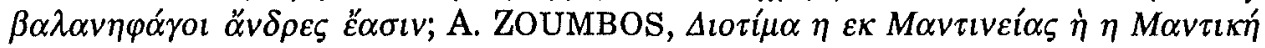
rvví ;

Du 11 au 13 novembre 1988 s'est tenu, à Compiègne, un colloque organisé par la Société de recherche interdisciplinaire "L'homme et l'animal" sur le thème Animal et pratiques religieuses : les manifestations matérielles. Trois communications concernaient la religion grecque antique : J. BERTIER, Le thème de l'abstinence de chair animale dans la philosophie ancienne; L. BODSON, L'offrande aux divinités grecques de l'effigie des animaux; J. LOPEZ-FEREZ, Animal et pratiques religieuses chez Euripide. Les Actes du colloque paraîtront, en 1989, dans un numéro spécial d'Anthropozoologica. Renseignements : Société "L'homme et l'animal», 55, rue Buffon, F-75005 PARIS.

En novembre 1988 s'est tenu, à Pise, à l'initiative du professeur R. TURCAN, un colloque sur le thème Mythe grec et art romain. 
Du 12 au 14 décembre 1988 s'est tenu, à l'Université de Sienne, un colloque intitulé La maschera, il doppio e il ritratto : strategie dell'identità. Communications de : M. BETTINI, G.B. BRONZINI, C. CALAME, A. FrASCHETTI, F. FRONTISI, G. GALlini, G. GUIDORIzZI, E. PELlizer, G. PUCCI, J.-P. VERNANT. Secrétariat : Facoltà di Lettere e Filosofia, 47, Via Roma, I - 53100 SIENA.

Les 27 et 28 janvier 1988 s'est tenu, à la Ruprecht-Karls-Universität de Heidelberg, le IIe Colloque de Sciences religieuses dont le thème était Inner und Außen : zur Religionsgeschichte des Inneren Menschen. Renseignements : Prof. Jan ASsMANN, Wissenschaftlich-Theologisches Seminar, Kisselgasse 1, D-6900 HEIDELBERG.

\section{Rencontres annoncées}

Du 8 au 10 mars 1989 se tiendra, à l'Université libre de Bruxelles, le Ier Congrès européen de Philosophie organisé par le Centre de philosophie ancienne de l'U.L.B. et la Société belge de Philosophie. Parmi les communications annoncées, on retiendra comme pouvant intéresser le domaine antique : H.G. GADAMER, La conscience herméneutique, condition de la compréhension phénoménologique de la pensée grecque et M. RICHIR, Pour une herméneutique phénoménologique de la pensée mythique. Organisation : L. COULOUBARITSIS et R. LEGROS, Société belge de Philosophie, 143, av. Buyl, B-1050 BRUXELLES.

Eu 16 au 18 mars 1989 se tiendra, à l'Université de Lille III, un Congrès international sur le thème Sciences historiques, sciences du passé et nouvelles technologies d'information. Bilan et évaluation. Thèmes traités : la linguistique, l'archéologie, la papyrologie, l'épigraphie, la numismatique, l'information scientifique et technique : enjeux et perspectives. Dans la quarantaine de communications prévues, on relèvera celle de S. COLE (Illinois University, Chicago), Data Bases for the Study of Greek Religion. Organisation : Prof. G. LOSFELD, Credo, Univ. de Lille III, B.P. 149, F-59653 VILLENEUVE D'ASCQ Cédex.

Du 16 au 18 mars 1989 se tiendra, à Liège, le IIe Colloque international organisé par le Centre d'Étude de la Religion Grecque Antique (Athènes) sur 
le thème Oracles et mantique en Grèce ancienne. Organisation : Prof. A. MOTTE et R. LAFFINEUR, 32, place du XX-Août, B-4000 LIÈGE. Pour le programme détaillé et les Actes de ce colloque, voir ci-après la rubrique «Prochains numéros de Kernos».

Du 16 au 18 avril 1989 se tiendra, à l'Université de Genève, un colloque international en l'honneur de Jean RUDHARDT sur le thème L'Orphisme et Orphée. Communications de Ph. BORGEAUD, J. BOTTERO, L. BRISSON, W. BURKERT, C. CALAME, G. CASADIO, M. DETIENNE, J. DÖRIG, F. GRAF, A. HURST, A. MONNIER, J. REDFIELD, J. RUDHARDT, D. SABBATUCCI, M. SCHMIDT, J. VINOGRADOV. Organisation : Prof. Ph. BORGEAUD, Département des Sciences de l'Antiquité, Univ. de Genève, CH-1211 GENÈVE 4.

Du 29 au 31 mai 1989 se tiendra, à Bruxelles et à Liège, un colloque organisé par le Groupe de contact interuniversitaire d'Études chypriotes sur le thème Les terres cuites chypriotes. Plusieurs communications traiteront d'aspects religieux, notamment des offrandes votives. Organisation : Prof. F. VANDENABEELE, Vrije Universiteit Brussels, Pleinlaan 2, B-1050 BRUSSEL et Prof. R. LAFFINEUR, Université de Liège, 32, Place du XX-Août, B-4000 LIÈGE.

Du 24 au 30 août 1989 se tiendra, à Pise, le IXe Congrès de l'International Federation of the Societies of Classical Studies (F.I.E.C.).

Du 1er au 6 septembre 1989 se tiendra, à Pérouse, le Ile Symposium platonicum. Le colloque a lieu sur invitation et sera consacré au Phèdre de Platon. Organisation : Prof. L. ROSSETTI, Istituto di Filosofia, Università di Perugia, I-06100 PERUGIA PG.

Du 14 au 15 septembre 1989 se tiendra, à l'Academia Belgica de Rome, une table ronde intitulée Héraclès, d'une rive à l'autre de la Méditerranée. Bilan et perspectives. Communications annoncées, le vendredi : F. BADER, Héraclès. De la préhistoire à l'idéologie tripartite; C. BAURAIN, Héraclès 
dans l'épopée homérique; P. LÉVÊQUE, Titre à communiquer; C. SEGAL, Héraclès chez Bacchylide, Stésichore et Pindare; W. BURKERT, Eracle e gli altri eroi culturali del vicino oriente; A. HERMARY, L'influence procheorientale dans l'élaboration de l'iconographie d'Héraclès; C. BONNET, Héraclès en Orient : interprétations et syncrétismes; le samedi : M. DETIENNE, Héraclès et Apollon; M. PIÉRART, Les honneurs de Persée et d'Héraclès; M. JOST, Héraclès en Arcadie; C. JOURDAIN-ANNEQUIN, Héraclès en Occident; M. LEGLAY, Héraclès-Hercule en Afrique du Nord; D. SABBATUCCI, Eracle ed Ercole. Cette table ronde est organisée à l'occasion du cinquantenaire de l'Academia Belgica, en hommage à Franz CUMONT, son premier directeur. Pour y être admis comme auditeur, il convient d'envoyer son inscription avant le 30 avril à C. BONNET, FNDP Namur, Dpt d'Histoire, rue de Bruxelles, 61, B-5000 NAMUR ou à C. JOURDAINANNEQUIN, Univ. des Sc. sociales de Grenoble II, U.E.R Histoire et histoire des Arts, Domaine universitaire, B.P. 25 X, F-38040 GRENOBLE.

Du 14 au 16 septembre 1989 se tiendra, à l'Université de Liège, un colloque international organisé par le Centre de recherches mythologiques de ParisNanterre et le Centre d'histoire des religions de l'Université de Liège sur le thème Mythe et Politique. Dans la vingtaine de communications prévues, plusieurs concerneront les mythes grecs et leurs survivances. Nombre limité de participants. Droit d'inscription : 1000 FB. Organisation : Prof. H. LIMET et A. MOTTE, Univ. de Liège, 32, place du XX-Août, B-4000 LIÈGE et Prof. F. JOUAN et B. DEFORGE, Univ. de Paris X, F-92001 NANTERRE Cédex.

Du 16 au 18 novembre 1989 se tiendra, à l'Université de Liège, le IXe Colloque organisé par le Groupe de Contact interuniversitaire d'Études phéniciennes et puniques sur le thème Phoinikeia Grammata (Aspects techniques, problèmes de diffusion et rôles de l'écriture). Organisation : Prof. C. BAURAIN, C. BONNET, V. KRINGS, 32, place du XX-Août, B-4000 LIÈGE.

Durant la dernière semaine d'avril 1990 se tiendra, à l'Université de Montpellier III, un colloque international organisé par le Séminaire d'étude des mentalités religieuses (S.E.M.A.) sur le thème L'initiation dans les cultures hellénique et romaine. Première circulaire disponible. Plus de trente communications sont déjà annoncées. Organisation : Prof. A. 
MOREAU et S. GELY, S.E.M.A., Univ. P. Valéry, B.P. 5043, F-34032 MONTPELLIER Cedex.

Du 3 au 9 septembre 1990 se tiendra, à Rome, le XVIIe Congrès de l'Association internationale pour l'histoire des religions (I.A.H.R.) sur le thème La notion de religion dans la recherche comparative. Parmi les quinze sections prévues, l'une est intitulée "Grèce et Rome. L'hellénisme». Droit d'inscription : 80 US \$. Les inscriptions pour une communication sont clôturées. Organisation : Prof. U. BIANCHI, Università di Roma «La Sapienza», 5, piazzale Aldo Moro, I-00185 ROMA.

En 1990, à l'occasion du 10e anniversaire de sa fondation, l'Association luxembourgeoise PRO LATINITATE se propose d'organiser, à Luxembourg, en collaboration avec les Universités de Liège et de Metz, un colloque sur le thème Parole sacrée, parole profane, de la religion à l'éloquence. Organisation : Mme R. KIEFFER, 10, rue Lemire, L-1927 LUXEMBOURG.

Fin août 1990 se tiendra, à Paris et à Istamboul, le IIe Congrès organisé par le Centre de recherches mythologiques de Paris-Nanterre et la Société de recherches psychothérapiques de langue française. Thème retenu : Le héros. Le congrès sera suivi d'un voyage en Asie Mineure. Renseignements: Prof. F. JOUAN, Université de Paris X, F-92001 NANTERRE Cédex.

\section{B. Expositions}

Durant l'été 1988 s'est tenue, à Lyon, une exposition organisée par le Musée archéologique de Lattes et l'Université P. Valéry de Montpellier sur le thème Les vases à mémoire. Collections de céramiques grecques du Midi de la France. Organisation : Christian LANDES et Annie-France LAURENS. Dans le catalogue on trouve notamment un chapitre intitulé "Survivances des dieux antiques, des héros et des hommes».

Du 17 au 23 février 1989 s'est tenue, à la Galerie de la Société Générale, Bd Tirou, à Charleroi (Belgique) une exposition de Christian LEROY intitulée Sculptures sur des thèmes de la mythologie grecque. Cette exposition est 
organisée par l'Association culturelle belgo-hellénique (Secrétariat : M. FAMELART, 22, av. H. Strauven, B-1160 BRUXELLES).

Du 13 au 31 mars 1989, dans le cadre du colloque international Oracles et mantique organisé à Liège par le C.E.R.G.A., se tiendra, à Liège, une exposition sur le thème Les sanctuaires oraculaires de Grèce. Organisation : Prof. R. LAFFINEUR, Univ. de Liège, 32, place du XX-Août, B-4000 LIÈGE.

\section{Conférences}

Le 19 octobre 1988, à l'invitation du Séminaire d'archéologie grecque de l'Université de Liège, Georges KORRES, professeur à l'Université d'Athènes, a fait une conférence sur La Pylos de Nestor : dix années de fouilles en Messénie mycénienne.

Le 8 novembre 1988, à l'invitation de l'Association luxembourgeoise Pro latinitate, André MOTTE, professeur à l'Université de Liège, a fait à la Bibliothèque nationale de Luxembourg, une conférence intitulée Le message religieux d'Éleusis. Le texte de cette conférence sera publié dans le Bulletin des Antiquités luxembourgeoises.

Le 20 janvier 1989, à l'invitation du Groupe de contact interuniversitaire d'Études chypriotes, W.-A. DASZEWSKI, professeur à l'Université de Varsovie et directeur du centre polonais d'archéologie méditerranéenne, a fait, à l'Université de Liège, une conférence intitulée Fouilles polonaises à Nea-Paphos.

Le 28 janvier 1989, à l'invitation de la Fédération des Professeurs de Grec et de Latin (Prés. : M.-L. DOCQUIER, 121/4, av. de l'Opale, B-1040 Bruxelles), Marcel DETIENNE, Directeur de la VIe Section à l'École pratique des Hautes Études de Paris, a fait, à l'Université de Liège, une conférence intitulée Les dieux dans la Cité grecque. 
Les 20 et 21 février 1989, à l'invitation de la Fondation archéologique de l'Université libre de Bruxelles et de la Vrije Universiteit, Pierre LÉVÊQUE, professeur à l'Université de Besançon, a fait deux conférences intitulées Imaginaire grec et imaginaire japonais et Le mythe de Pandore.

Le 23 février 1989, à l'invitation de la Société belge de Philosophie, Pierre HADOT, professeur au Collège de France, a fait, à l'Université de Liège, une conférence intitulée Ouranos, Kronos, Zeus, Aphrodite chez Plotin. 